\title{
Agro-morphological Variability Study of Barley (Hordeum vulgare L.) Landraces in Jumla, Nepal
}

\author{
Salik R. Gupta ${ }^{1}$, Madhusudan P. Upadhyay ${ }^{1}$ and Uma S. Shah ${ }^{2}$ \\ ${ }^{1}$ Agriculture Botany Division, NARC, Khumaltar, Lalitpur, Nepal \\ <gupta.salikram@yahoo.com> \\ ${ }^{2}$ Biotechnology Unit, NARC, Khumaltar, Lalitpur, Nepal
}

\begin{abstract}
Barley is an important winter cereal crop in Jumla (2240-3000 masl), Nepal. It is grown in different cropping patterns in both Khet and Bari land conditions. A total of 207 accessions of barley landraces collected from various locations of Jumla were studied for agromorphological characteristics in Khumaltar and Jumla. Data on eight qualitative and five quantitative traits in 2-m row plot were recorded to assess diversity among the landraces. Bhuwali, Chawali, Lekali and Pawai were four farmer-named traditional barley varieties under cultivation in Jumla. A range of variability was observed among the accessions of barley landraces. All accessions are six-row covered barley possessing rough awns and whitish-brown grains. These landraces exhibit a range of variation in growth class, spike density and rachilla hair length. Lekali and Pawai are adapted to the high-altitude area of Jumla. Cluster analysis of measured data under on-farm and on-station conditions indicated five distinct clusters. Three principal components explained $84.3 \%$ and $60.2 \%$ of the total variation from on-station and on-farm, respectively.
\end{abstract}

Key words: Hordeum vulgare, landraces, morphological variation, on-farm, on-station

\section{INTRODUCTION}

Barley (Hordeum vulgare L.) is one of the old and an important winter cereal crop for the people living in high mountain areas of Nepal. High level of barley diversity is observed in the Nepalese high lands and the region is considered a center of diversity for barley (Witcombe and Gilani 1979). Barley is cultivated in a wide range of environments in Nepal (Baniya et al 1997). Although, the acreage of barley in Nepal is limited, it is very important crop in remote and food deficit areas. Jumla is a transition zone between lower elevations, where a summer crop follow a winter cereal, and higher elevations where only one crop (crop is grown in summer season only) can be grown. Barley is the second cereal crop to rice in Jumla. It is utilized as food, beverages and livestock feed and also served as an indicator of well-being in the community of remote areas as the crop tolerates cold climate.

Morphological characterization is the foundation of genetic diversity research at any taxonomic level (Chandran and Pandya 2000). It is still an important tool for the management of crop germplasm collections (Ariyo 1993, Polignano et al 1993, Annicchiarico and Pecetti 1994) having been used to identify duplicates, to discriminate among material from different geographic areas, to establish core collections, to investigate relationships between landraces and their wild, and to prioritize material for use in breeding programmes. 
Pradhanang and Sthapit (1995), Witcombe and Murphy (1986) and Konishi and Matsuura (1991) reported that high level of variability was observed within and among populations. In Jumla, farmers classify their land according to its utilization and ecological conditions. The major land types are Khetland $^{1}\left(\right.$ Kholapane $^{8}$, Sim ${ }^{7}$, and Gadkule $\left.{ }^{6}\right)$, Bariland $^{2}$ (Gharbari ${ }^{5}$ and Pakhobari ${ }^{4}$ ) and Lekhland ${ }^{3}$. Barley is cultivated in Khet, Bari and Lekh. The amount of farmyard manure applied is normally between 17 and $27 \mathrm{t} \mathrm{ha}^{-1}$ for Khet and Bari. In Kehtland, fertilization is done through in situ manuring by the animal herds (Rana et al 2000). A more intensive cropping pattern was observed in Pakhobari. Gharbari is suitable for residence, kitchen garden and fruit orchards whereas mono cropping is practiced in Lekhland. The land types of the study site were $40 \%$ Khetland and $60 \%$ Bariland (Paudel et al 1998). The cropping patterns were observed in different land types. Ricebarley is the major cropping pattern in both Kholapane and Gadkule khet whereas rice-fallow is the major pattern in Simkhet. Barley-fallow cropping pattern is usually practiced in Lekhland. Similarly, barley-beans in Gharbari and barley-buckwheat, barley-beans, barley-proso millet cropping pattern is common in Pakhobari and it is considered as the most productive and very important land type with respect to food security.

Chawali, Bhuwali, Lekali and Pawai are four farmers' varieties of barley that are under cultivation in these land conditions for their preferred traits and different use values (Paudel et al 1998, Rana et al 2000). Among them, Chawali and Lekali are two very common landraces grown in large areas and by many farmers. Field study and household survey, indicates that Chawali was commonly cultivated in rice fields in winter where as Lekali is grown in diverse conditions from lowlands in rice field to Bari (upland) on hill tops and terraces under rainfed conditions. These varieties constitute a range of variability in agro-morphological traits and farmers use these traits as descriptors to identify and distinguish the varieties that have been maintained by them on-farm. The study was therefore aimed to measure the range of variation in agro-morphological characteristics among the barley accessions with distinct farmer units of diversity being maintained on-farm.

\section{MATERIALS AND METHODS}

A total of 207 accessions of barley landraces were collected from various locations of Jumla ranging from 2260 to $2530 \mathrm{~m}$ elevation. Collection was carried out from farmers' fields. Three to five ears from each hill were collected from field at the time of harvest. Each collection was considered as an accession and consisted of bulk of seeds of a particular population from farmers' plots (Table 1). These materials were evaluated on-station for morphological and agronomical variability under Khumaltar conditions $(1350 \mathrm{~m})$. Among 207 barley landrace populations only 96 were evaluated onfarm for their agro-morphological traits in farmers' fields by farmers' group in Jumla due to loss of other 111 accessions by insect damage during storage in the farmer's house. One set of these

\footnotetext{
${ }^{1}$ Khetland - bunded and irrigated land mainly for puddle rice.

${ }^{2}$ Bariland - unbunded and unirrigated upland.

${ }^{3}$ Lekhland - upland situated at high altitude far from village.

${ }^{4}$ Pakhobari - marginal and sloppy land located far from homestead devoted to neglected crops, grass and fodder.

${ }^{5}$ Gharbari - unbunded and unirrigated land around homestead with kitchen garden and orchard.

${ }^{6}$ Gadkule - Khet land irrigated from snow-melted river.

${ }^{7}$ Simkhet - water logged marshy land with poor drainage.

${ }^{8}$ Kholapane - Khet land irrigated by stream.
} 
materials with their passport information was supplied to Agriculture Botany Division for ex situ conservation.

Samples were planted in 2-m long, 2-row plots, with $25 \mathrm{~cm}$ spacing from row to row. The study was carried out under rainfed conditions with 30:30:0 NPK kg/ha fertilization before sowing and 15 $\mathrm{kg} / \mathrm{ha}$ of $\mathrm{N}_{2}$ was top-dressed at 2 months after planting. Data on quantitative traits (days to $50 \%$ heading, days to maturity, plant height, spikelet groups per spike, spike length, tiller number, effective tiller number and filled grains per head), and qualitative traits (growth class, row number, hoodedness, awn roughness, rachilla hair length, kernel covering and lemma colour) were recorded in five individual plants selected randomly using the IPGRI descriptors for barley (IPGRI 1994). Morphological traits were measured on 10 randomly selected plants of each accession in on-farm study in Jumla.

Descriptive statistics of both quantitative and qualitative traits were calculated by using the MS EXCEL program. In addition, Principal Component Analysis (PCA) and clustering methods using Unweighted Pair Group Method Analysis (UPGMA) were carried for quantitative traits on both sites and diversity indices using Shannon Weaver Index were calculated for qualitative traits. PCA allows reduction of a dimension problem. The techniques consist of reducing the structure and data matrix starting from a linear method and setting new variables called principal components. PCA and cluster analyses allowed identification of groups of objects or variables that are important in determining the amount of variability accounted by each of the axes. Both PCA and cluster analysis were performed using MINITAB-12 software.

Table 1. Number of accessions and collecting sites

\begin{tabular}{lrrrrr}
\hline \multirow{2}{*}{ Collecting site } & \multirow{2}{*}{ Altitude, $\mathrm{m}$} & \multicolumn{5}{c}{ Number of accessions } \\
\cline { 3 - 6 } & & Bhuwali & Chawali & Lekali & Pawai \\
\hline Bayalkatiya & $2500-2530$ & 5 & 8 & - & - \\
Damaibada & $2300-2320$ & - & 4 & - & - \\
Dhaulabada & $2320-2380$ & 1 & 13 & 1 & - \\
Kartikswami-8 & 2530 & - & 1 & - & - \\
Khalla & 2530 & 1 & 3 & 1 & - \\
Kotila & $2410-2480$ & 2 & 13 & - & - \\
Rokayabada & 2320 & 2 & 3 & - & - \\
Seridhuska & $2480-2500$ & 7 & 27 & - & 1 \\
Silam & 2530 & 1 & 4 & 1 & - \\
Talebhir & $2480-2520$ & 15 & 17 & - & - \\
Talium 1, 3-6, 8 and 9 & $2260-2500$ & 4 & 53 & 13 & - \\
Umgad & 2500 & 1 & 2 & - & - \\
\hline Total & & 42 & 148 & 16 & 1 \\
\hline
\end{tabular}

\section{RESULTS}

Simple statistical analysis of the observed data showed a range of variability among the accessions of barley landraces. These landraces showed variability in spike and grain morphological characters both on-farm and on-station. The differences with high range and diversity indices were observed in plant height $(40-74 \mathrm{~cm})$, spike characters such as row numbers (17.59), well filled grains per spike $(18-50)$ and $50 \%$ of flowering days (92-117) in quantitative traits. The landraces also showed 
variability in spike and grain morphological traits both on-farm and on-station (Table 2). The landraces were winter $(9.5 \%$ Bhuwali, $95.3 \%$ Chawali and $18.8 \%$ Lekali) and facultative types (78.6\% Bhuwali, 4.4\% Chawali and 81.3\% Lekali) except five samples (11.9\%) of Bhuwali, which has spring growth habit (Table 3 ). No variation was observed in row number, hoodedness, awn roughness, kernel covering and lemma colour. However, variation was observed in spike density and rachilla hair length. The Pawai landrace of barley had only one accession so its variability could not be measured.

Table 2a. Statistical parameters for quantitative traits of barley from on-station (Khumaltar)

\begin{tabular}{|c|c|c|c|c|c|c|}
\hline \multirow{3}{*}{ Characters } & \multicolumn{6}{|c|}{ On-station (Khumaltar) } \\
\hline & \multicolumn{2}{|c|}{ Bhuwali } & \multicolumn{2}{|c|}{ Chawali } & \multicolumn{2}{|r|}{ Lekali } \\
\hline & Range & $\mathrm{X} \pm \mathrm{SD}^{\dagger}$ & Range & $\mathrm{X} \pm \mathrm{SD}^{\dagger}$ & Range & $\mathrm{X} \pm \mathrm{SD}$ \\
\hline \multicolumn{7}{|l|}{ Phenological } \\
\hline $50 \%$ heading, $\mathrm{d}$ & $95-117$ & $111.7 \pm 7.7$ & $92-117$ & $96.35 \pm 4.7$ & $93-11$ & $7 \quad 109.6 \pm 9$. \\
\hline Maturity, d & $\begin{array}{r}139- \\
149\end{array}$ & $147.1 \pm 3.1$ & 139-149 & $144.7 \pm 3.8$ & $8 \quad 145-15($ & $0 \quad 148.3 \pm 1$ \\
\hline \multicolumn{7}{|l|}{ Morphological } \\
\hline$\overline{\text { Plant height, } \mathrm{cm}}$ & $40-72$ & $56.1 \pm 6.7$ & $36-74$ & $50.92 \pm 6.2$ & $45-7$ & $\begin{array}{ll}0 & 55.94 \pm 7 .\end{array}$ \\
\hline Spike length, cm & - & - & - & - & - & - \\
\hline Spikelet/spike, n & $20-59$ & $44.9 \pm 7.8$ & $17-58$ & $38.18 \pm 7.5$ & $28-5$ & $\begin{array}{ll}6 & 45.12 \pm 7 .\end{array}$ \\
\hline Tiller, $\mathrm{n}$ & - & - & - & - & - & - \\
\hline Effective tiller, $\mathrm{n}$ & - & - & - & - & - & - \\
\hline Filled grain/head, n & - & - & - & - & - & - \\
\hline$\underline{\text { Grain }}$ & & & & & & \\
\hline 1000-grain wt, g & $\begin{array}{r}36.4- \\
46\end{array}$ & $41.1 \pm 2.3$ & $24.8-479$ & $38.19 \pm 3.1$ & $36.8-4 c$ & $39.8 \pm 2$. \\
\hline \multicolumn{7}{|c|}{${ }^{\dagger} X \pm S D=$ Mean \pm Standard deviation. } \\
\hline \multicolumn{7}{|c|}{$\begin{array}{l}\text { Table } 2 \text { b. Statistical parameters for quantitative traits of barley from on-farm trial } \\
\text { (Jumla) }\end{array}$} \\
\hline \multirow{3}{*}{ Characters } & \multicolumn{6}{|c|}{ On-farm (Jumla) } \\
\hline & \multicolumn{2}{|c|}{ Bhuwali } & \multicolumn{2}{|c|}{ Chawali } & \multicolumn{2}{|c|}{ Lekali } \\
\hline & Range & $\mathrm{X} \pm \mathrm{SD}^{\dagger}$ & Range & $\mathrm{X} \pm \mathrm{SD}^{\dagger}$ & Range & $\mathrm{X} \pm \mathrm{SD}^{\dagger}$ \\
\hline $\begin{array}{l}\text { Maturity, d } \\
\text { Morphological }\end{array}$ & - & - & - & - & - & - \\
\hline Plant height, cm & $55-61$ & $57.5 \pm 2.3$ & $37.5-83$ & $60.8 \pm 10$ & $42.9-60$ & $53.4 \pm 9.2$ \\
\hline Spike length, cm & $4-5.4$ & $4.7 \pm 0.6$ & $2.6-7.1$ & $4.4 \pm 0.7$ & $2.2-4.8$ & $3.6 \pm 1.3$ \\
\hline Spikelet/spike, n & - & - & - & - & - & - \\
\hline Tiller, $\mathrm{n}$ & $2.6-4$ & $3.4 \pm 0.5$ & $1.8-8.5$ & $3.8 \pm 1.1$ & $2.4-3.9$ & $3.3 \pm 0.8$ \\
\hline Effective tiller, $\mathrm{n}$ & $1.6-3.2$ & $2.6 \pm 0.6$ & $1.1-6.1$ & $2.9 \pm 0.9$ & $1.9-2.7$ & $2.3 \pm 0.4$ \\
\hline Filled grain/head, n & $23-48$ & $37 \pm 10$ & $18.2-50$ & $33.1 \pm 7.2$ & $26.6-37$ & $30.9 \pm 5.7$ \\
\hline$\underline{\text { Grain }}$ & & & & & & \\
\hline 1000-grain wt, g & $33-43$ & $39.1 \pm 4.2$ & $27-47.2$ & $38.6 \pm 3.4$ & $36-43.4$ & $40.7 \pm 4.1$ \\
\hline
\end{tabular}


A pairwise association among landraces of barley (on-station and on-farm) was measured from the observed agro-morphological traits using Ward and Euclidean distance and revealed a clear clustering into different morphological groups (Table 4). The resulting dendrograms depicted in Figures 1 and 2 show the relationship between populations of barley landraces and grouped all populations into five distinct clusters for both on-station and on-farm characterizations respectively. In both clusterings, Chawali landraces were found to be most diverse and represented in all the clusters (Table 5). However, Bhuwali and Lekali landrace populations predominantly represented cluster II. The study showed the distribution of landraces in different clusters irrespective of farmers' given name and their descriptions.

Table 3. Frequency distribution and Shannon Weaver indices of qualitative traits of barley (on-station)

\begin{tabular}{|c|c|c|c|c|c|c|c|c|c|c|c|}
\hline \multirow[b]{2}{*}{ Character } & \multicolumn{3}{|c|}{ Bhuwali } & \multicolumn{3}{|c|}{ Chawali } & \multicolumn{3}{|c|}{ Lekali } & \multicolumn{2}{|c|}{ Pawai } \\
\hline & $\begin{array}{c}\text { No. } \\
\text { of } \\
\text { acc. }\end{array}$ & $\begin{array}{c}\text { Freq. } \\
\%\end{array}$ & $\mathrm{H}$ & $\begin{array}{c}\text { No. } \\
\text { of } \\
\text { acc. }\end{array}$ & $\begin{array}{c}\text { Freq. } \\
\%\end{array}$ & $\mathrm{H}$ & $\begin{array}{c}\text { No. } \\
\text { of } \\
\text { acc. }\end{array}$ & $\begin{array}{c}\text { Freq. } \\
\%\end{array}$ & $\mathrm{H}$ & $\begin{array}{c}\text { No. } \\
\text { of } \\
\text { acc. }\end{array}$ & $\begin{array}{c}\text { Freq. } \\
\%\end{array}$ \\
\hline \multicolumn{12}{|l|}{ Growth class } \\
\hline Winter & 4 & 9.5 & \multirow{3}{*}{0.67} & 141 & 95.3 & \multirow{3}{*}{0.19} & 3 & 18.8 & \multirow{3}{*}{0.48} & 1 & 100 \\
\hline Facultative & 33 & 78.6 & & 7 & 4.7 & & 13 & 81.3 & & 0 & 0 \\
\hline Spring & 5 & 11.9 & & 0 & 0 & & 0 & 0 & & 0 & 0 \\
\hline \multicolumn{12}{|l|}{ Row number } \\
\hline Six row & 42 & 100 & \multirow{2}{*}{0} & 148 & 100 & \multirow{2}{*}{0} & 16 & 100 & \multirow{2}{*}{0} & 1 & 100 \\
\hline Two row & 0 & 0 & & 0 & 0 & & 0 & 0 & & 0 & 0 \\
\hline \multicolumn{12}{|l|}{ Spike density } \\
\hline Lax & 38 & 90.5 & \multirow{3}{*}{0.31} & 95 & 64.2 & \multirow{3}{*}{0.65} & 14 & 87.5 & \multirow{3}{*}{0.38} & 1 & 100 \\
\hline Intermediate & 4 & 9.5 & & 53 & 35.8 & & 2 & 12.5 & & 0 & 0 \\
\hline Dense & 0 & 0 & & 0 & 0 & & 0 & 0 & & 0 & 0 \\
\hline \multicolumn{12}{|l|}{ Hoodedness } \\
\hline Sessile hoods & 0 & 0 & \multirow{4}{*}{0} & 0 & 0 & \multirow{4}{*}{0} & 0 & 0 & \multirow{4}{*}{0} & 0 & 0 \\
\hline Elevated hoods & 0 & 0 & & 0 & 0 & & 0 & 0 & & 0 & 0 \\
\hline Awnless & 0 & 0 & & 0 & 0 & & 0 & 0 & & 0 & 0 \\
\hline Awned & 42 & 100 & & 148 & 100 & & 16 & 100 & & 1 & 100 \\
\hline \multicolumn{12}{|l|}{ Awn roughness } \\
\hline Smooth & 0 & 0 & \multirow{2}{*}{0} & 0 & 0 & \multirow{2}{*}{0} & 0 & 0 & \multirow{2}{*}{0} & 0 & 0 \\
\hline Rough & 42 & 100 & & 148 & 100 & & 16 & 100 & & 1 & 100 \\
\hline \multicolumn{12}{|l|}{ Rachilla hair length } \\
\hline Short & 24 & 57.1 & \multirow{2}{*}{0.68} & 125 & 84.5 & \multirow{2}{*}{0.43} & 14 & 87.5 & & 1 & 100 \\
\hline Long & 18 & 42.9 & & 23 & 15.5 & & 2 & 12.5 & 0.69 & 0 & 0 \\
\hline Kernel covering & & & & & & & & & & & \\
\hline Naked grains & 0 & 0 & & 0 & 0 & & 0 & 0 & & 0 & 0 \\
\hline Covered grains & 42 & 100 & 0 & 148 & 100 & 0 & 16 & 100 & 0 & 1 & 100 \\
\hline Lemma colour & & & & & & & & & & & \\
\hline White/brown & 42 & 100 & & 148 & 100 & & 16 & 100 & & 1 & 100 \\
\hline Purple or black & 0 & 0 & 0 & 0 & 0 & 0 & 0 & 0 & 0 & 0 & 0 \\
\hline Pink & 0 & 0 & & 0 & 0 & & 0 & 0 & & 0 & 0 \\
\hline
\end{tabular}


Table 4. Means of relevant quantitative traits for two sites of the clusters formed

\begin{tabular}{|c|c|c|c|c|c|c|c|c|c|c|c|c|}
\hline \multirow{3}{*}{ Character } & \multicolumn{12}{|c|}{ Clusters } \\
\hline & \multicolumn{6}{|c|}{ On-station } & \multicolumn{6}{|c|}{ On-farm } \\
\hline & I & II & III & IV & V & Mean & I & II & III & IV & V & Mean \\
\hline $\begin{array}{l}50 \% \\
\text { heading, } \mathrm{d}\end{array}$ & 94.8 & 111.1 & 95.8 & 95.2 & 95.6 & 100.6 & - & - & - & - & - & - \\
\hline $\begin{array}{l}\text { Plant height, } \\
\mathrm{cm}\end{array}$ & 50.2 & 58.2 & 44.5 & 55.5 & 52.4 & 52.4 & 56.6 & 52.6 & 63.4 & 61.5 & 63.4 & 61.0 \\
\hline $\begin{array}{l}\text { Spike length, } \\
\mathrm{cm}\end{array}$ & - & - & - & - & - & - & 4.0 & 4.5 & 4.3 & 4.0 & 4.6 & 4.3 \\
\hline $\begin{array}{l}\text { Spikelet } \\
\text { groups/spike, } \\
\text { n }\end{array}$ & 46.4 & 46.5 & 35.7 & 37.9 & 29.3 & 40.2 & - & - & - & - & - & - \\
\hline Tiller, $\mathrm{n}$ & - & - & - & - & - & - & 3.2 & 4.1 & 3.9 & 3.6 & 3.8 & 3.8 \\
\hline $\begin{array}{l}\text { Effective } \\
\text { tiller, } n\end{array}$ & - & - & - & - & - & - & 2.5 & 3.0 & 2.9 & 2.7 & 2.8 & 2.8 \\
\hline $\begin{array}{l}\text { Filled } \\
\text { grain/head, n }\end{array}$ & - & - & - & - & - & - & 32.6 & 30.3 & 35.0 & 35.4 & 33.5 & 33.8 \\
\hline $\begin{array}{l}\text { Unfilled } \\
\text { grain/head }\end{array}$ & - & - & - & - & - & - & 2.4 & 1.9 & 1.9 & 1.8 & 1.8 & 1.9 \\
\hline Maturity, d & 139.0 & 148.3 & 144.9 & 147.1 & 148.0 & 145.6 & - & - & - & - & - & - \\
\hline $\begin{array}{l}\text { 1000-grain } \\
\text { weight, g }\end{array}$ & 38.9 & 41.2 & 37.4 & 38.1 & 38.2 & 38.9 & 37.0 & 37.7 & 39.1 & 38.7 & 39.2 & 38.7 \\
\hline
\end{tabular}

Table 5. Composition and characteristics of clusters based on observed traits for two locations

\begin{tabular}{|c|c|c|c|c|c|c|c|c|}
\hline \multirow[b]{2}{*}{ Cluster } & \multicolumn{4}{|c|}{ On-station } & \multicolumn{4}{|c|}{ On-farm } \\
\hline & $\begin{array}{l}\text { No. of } \\
\text { acc. }\end{array}$ & $\begin{array}{l}\text { Landrace } \\
\text { name }\end{array}$ & $\begin{array}{l}\text { Total } \\
\text { no. } \\
\text { types }\end{array}$ & $\begin{array}{l}\text { Cumulative } \\
\text { characteristics of } \\
\text { landraces in cluster }\end{array}$ & $\begin{array}{l}\text { No. of } \\
\text { acc. }\end{array}$ & $\begin{array}{l}\text { Landrace } \\
\text { name }\end{array}$ & $\begin{array}{l}\text { Total } \\
\text { no. } \\
\text { types }\end{array}$ & $\begin{array}{l}\text { Cumulative } \\
\text { characteristics of } \\
\text { landraces in } \\
\text { cluster } \\
\end{array}$ \\
\hline I & 39 & $\begin{array}{l}\text { Bhuwali } \\
\text { (4) and } \\
\text { Chawali } \\
(35)\end{array}$ & 2 & $\begin{array}{l}\text { Early heading \& } \\
\text { maturity, medium } \\
\text { plant height \& } \\
\text { more no. of } \\
\text { spikelet groups per } \\
\text { spike }\end{array}$ & 8 & $\begin{array}{l}\text { Bhuwali } \\
\text { (1), and } \\
\text { Chawali } \\
\text { (7) }\end{array}$ & 2 & $\begin{array}{l}\text { Medium plant } \\
\text { height, short } \\
\text { spike, low } \\
\text { tillering and low } \\
\text { 1000-seed wt. }\end{array}$ \\
\hline II & 55 & $\begin{array}{l}\text { Bhuwali } \\
\text { (34), } \\
\text { Chawali } \\
(8), \\
\text { Lekali } \\
\text { (12) and } \\
\text { Pawai (1) }\end{array}$ & 4 & $\begin{array}{l}\text { Late heading \& } \\
\text { maturity, taller } \\
\text { plant and high } \\
\text { number of spikelet } \\
\text { groups/spike and } \\
\text { 1000-grain weight }\end{array}$ & 14 & $\begin{array}{l}\text { Bhuwali } \\
\text { (11), } \\
\text { Chawali } \\
\text { (2) and } \\
\text { Unknown } \\
\text { (1) }\end{array}$ & 2 & $\begin{array}{l}\text { Dwarf plant, long } \\
\text { spike, high } \\
\text { tillering and } \\
\text { medium 1000- } \\
\text { seed wt. }\end{array}$ \\
\hline III & 45 & $\begin{array}{l}\text { Bhuwali } \\
(3) \text {, } \\
\text { Chawali } \\
\text { (40) and } \\
\text { Lekali (2) }\end{array}$ & 3 & $\begin{array}{l}\text { Dwarf plant, } \\
\text { medium maturity } \\
\text { \& heading and low } \\
\text { seed weight }\end{array}$ & 36 & $\begin{array}{l}\text { Bhuwali } \\
\text { (2), } \\
\text { Chawali } \\
\text { (33) and } \\
\text { Unknown } \\
\text { (1) }\end{array}$ & 2 & $\begin{array}{l}\text { Tall plant, } \\
\text { medium tiller \& } \\
\text { spike length, } \\
\text { moderately high } \\
\text { number of grain } \\
\text { per head }\end{array}$ \\
\hline IV & 40 & $\begin{array}{l}\text { Chawali } \\
\text { (38) and } \\
\text { Lekali (2) }\end{array}$ & 2 & $\begin{array}{l}\text { Medium heading, } \\
\text { plant height, } \\
\text { maturity \& } 1000- \\
\text { seed weight }\end{array}$ & 15 & $\begin{array}{l}\text { Chawali } \\
\text { (15) }\end{array}$ & 1 & $\begin{array}{l}\text { Medium plant, } \\
\text { short spike, with } \\
\text { medium tillering } \\
\text { and high number } \\
\text { of filled } \\
\text { grain/head. }\end{array}$ \\
\hline $\mathrm{V}$ & 28 & $\begin{array}{l}\text { Bhuwali } \\
\text { (2), } \\
\text { Chawali } \\
(26)\end{array}$ & 2 & $\begin{array}{l}\text { Low seed weight \& } \\
\text { number of spikelet } \\
\text { groups/spike and } \\
\text { medium plant } \\
\text { height \& maturity. }\end{array}$ & 23 & $\begin{array}{l}\text { Bhuwali } \\
\text { (1), } \\
\text { Chawali } \\
\text { (21) and } \\
\text { Unknown } \\
\text { (1) }\end{array}$ & 2 & $\begin{array}{l}\text { Tall plant, long } \\
\text { spike, medium } \\
\text { tiller number but } \\
\text { higher } 1000 \text {-seed } \\
\text { wt. }\end{array}$ \\
\hline
\end{tabular}


Principal component analysis also showed a marked variation among landraces with great influence of the morphological traits of plant, spikes and grains along the first three axes (Table 6). Figure 3 shows the distribution of barley populations by landrace names under the on-station characterization. Three PCs accounted $84.3 \%$ of the total variance of five quantitative characters measured from on-station, and $60.2 \%$ of total variance of seven quantitative characters measured from the on-farm trial (Table 6). The first principal component explained $46.4 \%$ and $29.5 \%$ of the total variance on-station and on-farm, respectively. Eigen values and eigenvectors were different between on-farm and on-station trials. The variation shown between two locations may be due not only to the location difference, but probably also to the differences in number of traits and accessions.

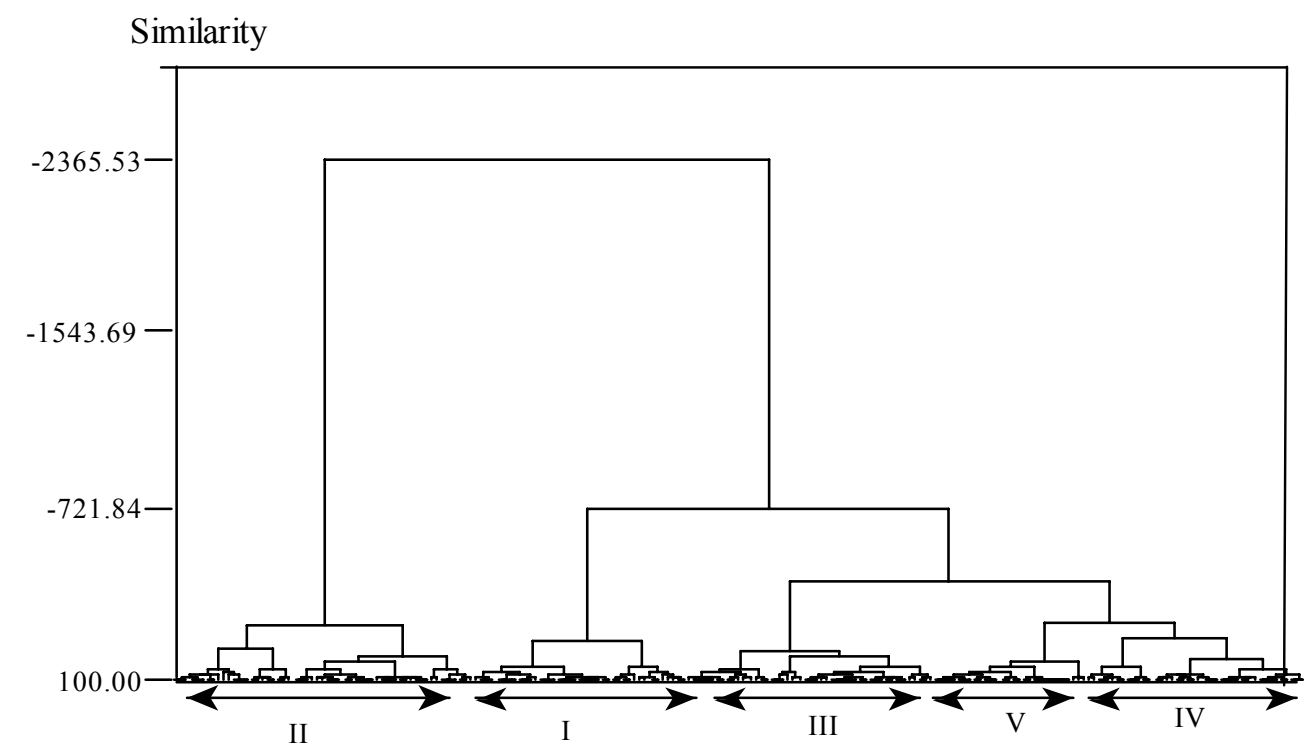

Figure 1. Dendrogram of 207 barley accessions revealed by UPGMA cluster analysis (on-station). 


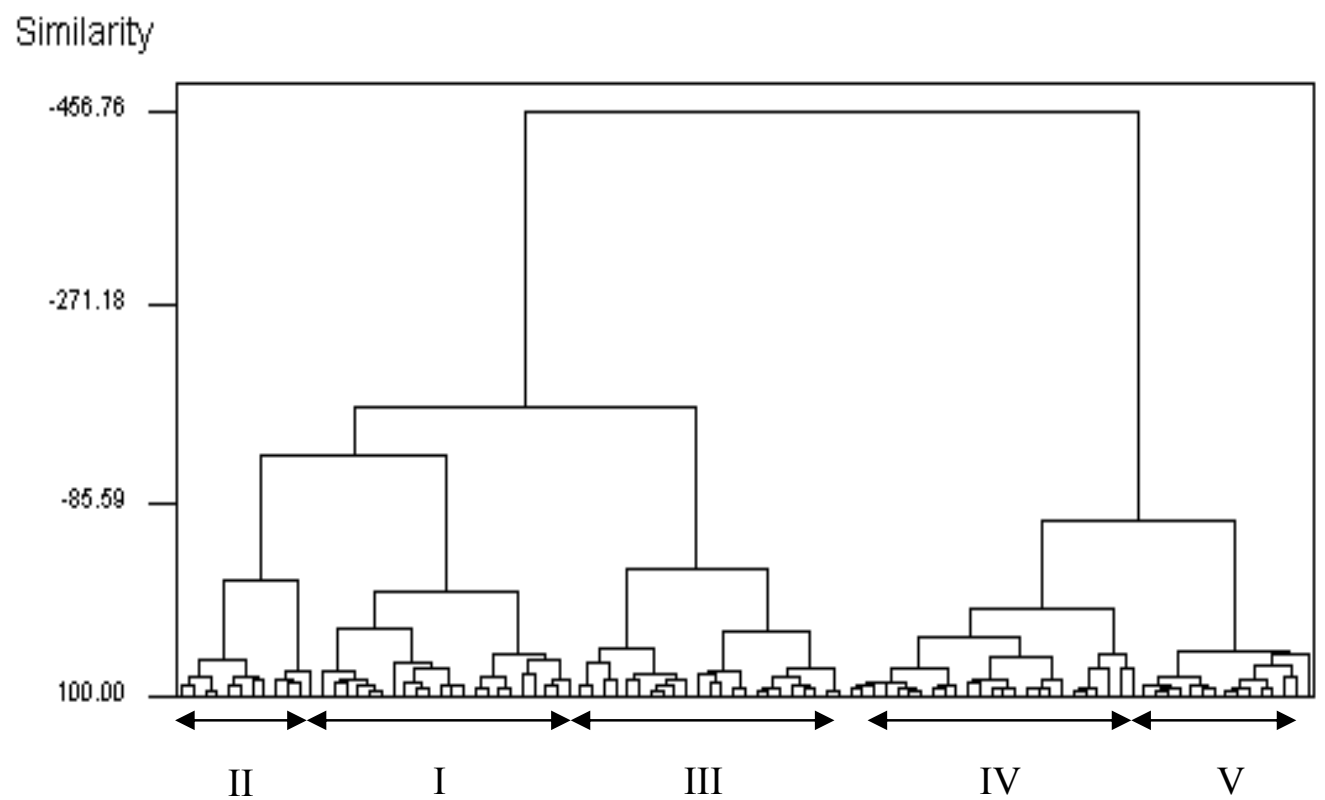

Figure 2. Dendrogram of 96 barley accessions revealed by UPGMA cluster analysis (on-farm).

Table 6. Eigenvectors, eigen values, total variance and cumulative variance for quantitative traits in barley landraces over two sites

\begin{tabular}{|c|c|c|c|c|c|c|}
\hline \multirow[t]{2}{*}{ Character } & \multicolumn{3}{|c|}{ On-station (Khumaltar) } & \multicolumn{3}{|c|}{ On-farm (Jumla) } \\
\hline & PC1 & PC2 & PC3 & PC1 & PC2 & PC3 \\
\hline $50 \%$ heading, $\mathrm{d}$ & -0.577 & 0.013 & 0.139 & - & - & - \\
\hline Plant height, $\mathrm{cm}$ & -0.482 & -0.122 & 0.529 & 3.94 & -0.234 & -0.173 \\
\hline Spike length, cm & - & - & - & 0.379 & 0.070 & 0.372 \\
\hline $\begin{array}{l}\text { Spikelet } \\
\text { groups/spike, n }\end{array}$ & -0.327 & 0.712 & 0.172 & - & - & - \\
\hline Tiller number & - & - & - & 0.505 & 0.264 & -0.281 \\
\hline Effective tiller, $n$ & - & - & - & 0.495 & 0.262 & -0.355 \\
\hline Filled grain/head, $n$ & - & - & - & 0.230 & -0.564 & -0.030 \\
\hline Unfilled grain/head & - & - & - & -0.131 & -0.371 & -0.183 \\
\hline Maturity, d & -0.355 & -0.679 & -0.070 & - & - & - \\
\hline 1000-grain weight, g & -0.449 & 0.131 & -0.816 & 0.239 & -0.379 & 0.278 \\
\hline Eigen values & 2.320 & 1.221 & 0.673 & 2.653 & 1.662 & 1.098 \\
\hline$\%$ of total variance & 46.4 & 24.4 & 13.5 & 29.5 & 18.5 & 12.2 \\
\hline $\begin{array}{l}\% \text { of cumulative } \\
\text { variance }\end{array}$ & 46.4 & 70.8 & 84.3 & 29.5 & 48.0 & 60.2 \\
\hline
\end{tabular}




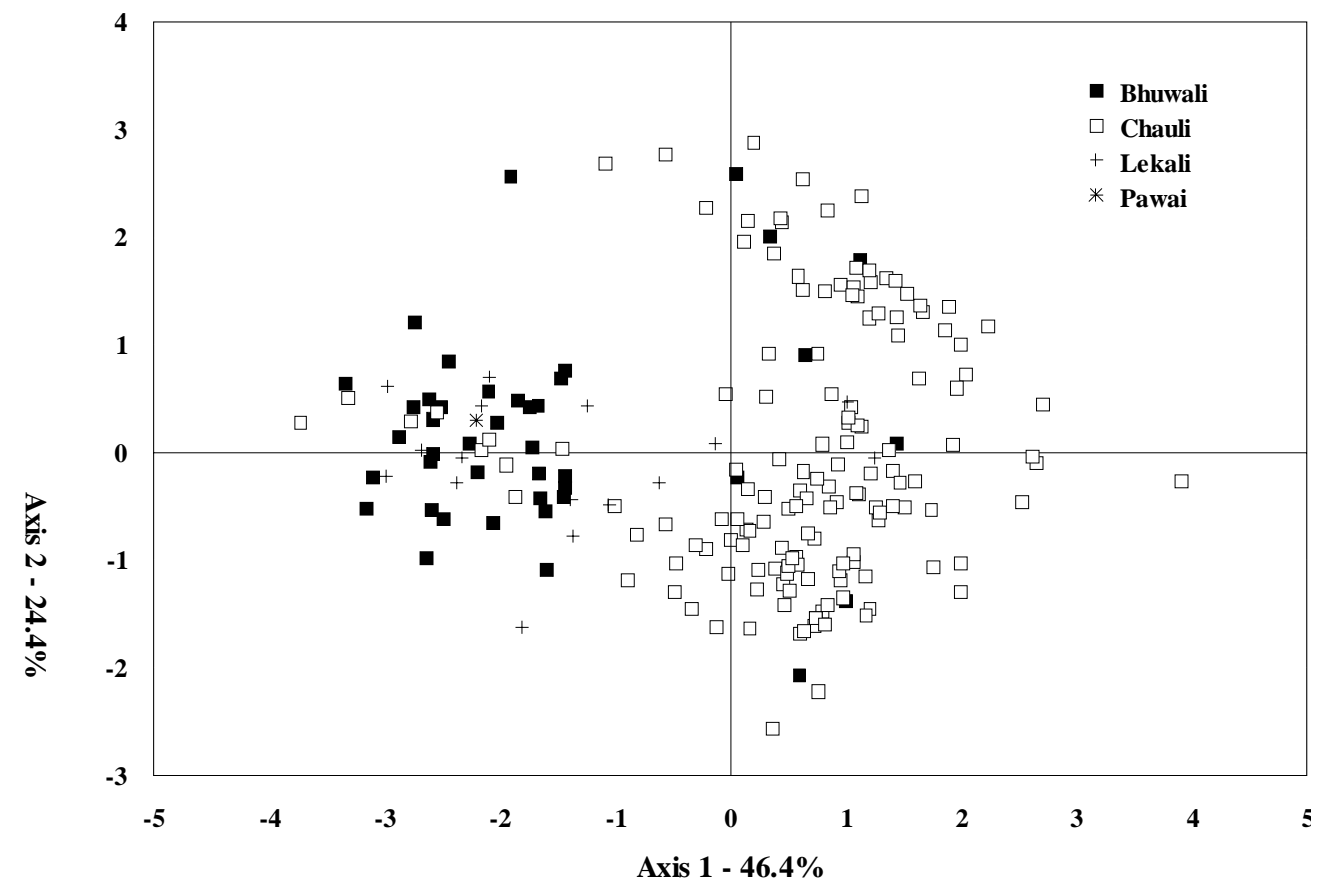

Figure 3. Distribution plot of barley landraces along with the first and second principal components (PCs).

Unlike to the hierarchical clustering, the principal component analysis of quantitative traits revealed a clear grouping of barley populations by landrace names. The accessions of Bhuwali and Chawali landraces clustered together and separated by the axis 2 with few outlier accessions (Figure 3 ).

Figure 6 also indicates that these landrace populations were most variable and were encompassed of range of intra-varietal variations for the observed quantitative traits.

\section{DISCUSSION}

A large amount of agro-morphological diversity was observed in the barley landraces belonged to four differently named landraces that were under cultivation in Jumla. Morphological diversity of quantitative traits between the three groups of barley landraces under study showed greater variation in both on-farm and on-station (Table 2). Chawali and Bhuwali landraces is grown in irrigated and well managed conditions and Lekali landraces is grown in rainfed and diverse conditions. Similarly, in qualitative traits also variability was observed in growth class, spike density and rachilla hair length (Table 3). The existence of considerable level of diversity in terms of isozyme variability among farmers' barley populations of differently named landraces. Bhuwali, Chawali, and Lekali consisted greater variation among populations and among farmers named varieties (Bajracharya et al 2001). These landraces can be good source materials for breeding purpose. Hence, it could be a valuable resource to conserve and for utilization with improvement in future.

The analysis of average value of the variables for each group provides information for describing the groups identified. High mean were observed for $50 \%$ heading and maturity on on-station and plant 
height for on-farm indicating that wide variation exists in the population for these traits (Table 4). No variation was observed in grain weight for both on-farm and on-station.

Table 5 gives a general description of each group. The studied populations clustered into five clusters with two sets of observation. In the clustering, Chawali landraces were found to be diverse and represented in all the clusters (Table 5). However, Bhuwali represented in cluster I and III whereas Lekali represented cluster II and IV (Figure 3). It suggests that there is presence of variability in the barley landraces. This study showed the distribution of landraces in different cluster irrespective of farmers' given name and their description. The structure and level of morphological variation revealed in the present study showed a significant level of genetic diversity among the barley accessions of differently named landraces. To an extent, the diverse morphological forms established the genetic identity of the barley landraces.

The studies on landraces have shown the value and importance of agro-morphological characters with direct relevance to local farmers and breeders for conservation, and in estimating diversity of germplasm and in describing the level of discrimination of the varieties (Murphy and Witcombe 1981 have also shown how farmers select preferred maize types from their agro-morphological characters. Similarly, Chu et al (1997) have shown how combining ethno-botanical methods with genetic analysis can give insights into how crop genetic diversity is maintained and managed. These descriptive values of morphological characters are genetically heritable and therefore worthy for the genetic diversity analysis. The first three principal components with eigen value greater than unity explained $84.3 \%$ and $60.2 \%$ on-station and on-farm of the total variation among the studied landraces for the quantitative traits (Table 6). The first and second principal components accounted for $46.4 \%$ and $70.8 \%$ on on-station and $29.5 \%$ and $48.0 \%$ on on-farm respectively of the total variation. 1000-grain weight, grain length and width were the most important traits contributing to the first principal components. Plant height, tiller number, panicle length and grain weight were the important traits contributing to the second and third principal component. Scattered plots of these landraces clearly indicated that there is presence of variability.

Barley landraces were identified according to their group in the dendrogram and graphed in biplots using the first three principal components (Figures 1 and 2). Based on the plots constructed using the first two principal components, groups I, II and III were spatially observed as distinct groups on both the sites while the IV axis was useful for better differentiation of group V.

From the analysis of the groups, the clustering pattern obtained showed an association with the agronomic performance of the accessions. This indicates that the classification can assist in discriminating the groups of material, which can be of future use for plant breeding programme.

This work has been a contribution to increase the knowledge about the barley germplasm conservation in Jumla, Nepal. This better understanding should allow a better conservation and use of the collection in breeding programmes. The research will also assist in the conservation of valuable germplasm, as is the case of local varieties, which hold important local adaptation and are of widespread use by farmers throughout the entire country.

\section{ACKNOWLEDGEMENTS}

The authors wish to express sincere thanks to the Chief of Agriculture Botany Division for providing necessary support and valuable suggestions during the experiment implementation. We thank Mr BK Joshi, Scientist, for technical support and data analysis. We also thank Mr DM Dongol and Mr RP Paudel for their hard work during data recording and compilation. International Plant Genetic Resources Institute (IPGRI) 
Nepal Agric. Res. J. Vol. 9, 2009

Project, Strengthening the Scientific Basis of In-situ Conservation of Agricultural Biodiversity on-farm supported this work.

\section{REFERENCES}

Annicchiarico P and L Pecetti. 1994. Morpho-physiological traits as descriptors for discrimination of durum wheat germplasm. Genet. Resour. Crop Evol. 41:47-54.

Ariyo OJ. 1993. Genetic diversity in West African okra (Abelmoschus caillei (A. Chev.) Stevels): Multivariate analysis of morphological and agronomic characteristics. Genet. Resour. Crop Evol. 40:25-32.

Bajracharya J, PR Tiwari, DM Shakya, BK Baniya and BR Sthapit. 2001. Farmer management and isozyme variation in barley landraces (Hordeum vulgare L.), Jumla, Nepal. In: On farm management of agricultural biodiversity in Nepal (BR Sthapit, MP Upadhyay, BK Baniya, A Subedi and BK Joshi, eds), 2003. Proceedings of a National workshop, NARC, LI-BIRD and IPGRI.

Baniya BK, DMS Dongol and KW Riley. 1997. Characterization of Nepalese barley germplasm. Rachis, Barley and wheat Newsletter, ICARDA, Aleppo, Syria. Vol.16, No 16. Pp. 16-19.

Chandran K and SM Pandya 2000. Morphological characterization of Arachis species of section Arachis. Plant Genet. Resour. Newslett. 121:38-41.

Chu Xu-Jian, Li X, Li R, Xu JC, Li XX and Li RG. 1997. Ethnobotany and genetic diversity of taro. IPGRI News I. For Asia, the Pacific and Oceania. IPGRI, Rome, Italy. 23:9-10.

IPGRI. 1994. Descriptors for barley (Hordeum vulgare L.). International Plant Genetic Resources Institute, Rome, Italy.

Konishi T and S Matsuura. 1991. Geographic differentiation in isozyme genotypes of Himalayan barely (Hordeum vulgare L.). Genome 34:704-709.

Murphy PJ and JR Witcombe. 1981. Variation in Himalayan barley and the concept of centres of diversity. In: Barley Genetics IV. Proceedings of the fourth international barley genetics symposium, Edinburgh. Pp. 26-36.

Paudel CL, PR Tiwari, JD Neupane and DP Devkota. 1998. Strengthening the scientific basis of in situ conservation of agrobiodiversity: Findings of site selection in Jumla, Nepal. NP working paper No 3/98. NARC/LI-BIRD-Nepal, IPGRI-Rome, Italy.

Polignano GB, P Uggenti and G Scippa. 1993. The patterns of genetic diversity in faba bean collections from Ethiopia and Afghanistan. Genet. Resour. Crop Evol. 40:71-75.

Pradhanang PM and BR Sthapit. 1995. Effect of cultivar mixtures on yellow rust incidence and grain yield of barley in the hills of Nepal. Crop Protection 14(4):331-334.

Rana RB, CL Paudel, PR Tiwari, D Gauchan, A Subedi, BR Sthapit, MP Upadhyay and DR Jarvis. 2000. In situ crop conservation: Findings of agroecological, crop diversity and socioeconomic base line survey of Talium ecosite, Jumla. NP working paper No 3/2000. NARC/LI-BIRD-Nepal, IPGRI-Rome, Italy.

Witcombe JR and MM Gilani. 1979. Variations in cereals from the Himalayas and the optimum strategy for sampling plant germplasm. Journal of Applied Ecology 16:633-640.

Witcombe JR and PJ Murphy. 1986. Covered and naked barelys from the Himalaya. 2. Why do they differ from each other so extensively? Theor. Appl. Genet. 71:736-741. 